\title{
Ultrasound screening for hip abnormalities: preliminary findings in 1001 neonates
}

\author{
LAURENCE BERMAN, LESLIE KLENERMAN
}

\begin{abstract}
Several studies have documented the failure of neonatal clinical screening to reduce the incidence of hip dislocation later in infancy. In addition, the practice of splinting unstable hips is said to result in the treatment of many infants who would have developed normally if left unsplinted. Ultrasound provides a detailed image of the bony and cartilaginous neonatal hip. The results of conventional testing for hip instability were compared with ultrasound screening in 1001 neonates. As a result of the ultrasonic image 14 of 17 infants with hip instability were not splinted and developed normally. Two babies without detectable clinical signs were shown to have severe hip abnormalities.

It is suggested that clinically normal but dysplastic hips do exist and that ultrasound will detect them. In addition, the overtreatment that is current practice might be avoided.
\end{abstract}

\section{Introduction}

It is assumed that established hip dislocation in infancy is a progression from the unstable neonatal hip. This association is unproved and has been questioned..$^{1-3}$ Because of this assumption, however, management has focused on the early detection of hip instability in the neonate, followed by treatment with splint or harness. It has been suggested that the splinting of unstable hips is misguided as most would resolve spontaneously ${ }^{1}$ and that dislocation may occur as a result of a clinically silent anatomical abnormality such as a shallow acetabulum..$^{1-3}$

Two widely practised tests for instability are the Ortolani test and the Barlow test. The first relies on a dislocated femoral head slipping into the acetabulum with a palpable "clunk." The second entails a further manoeuvre, whereby an attempt is made to dislocate the femur if the initial re-entry clunk is not elicited. This second part of

Northwick Park Hospital and Clinical Research Centre, Harrow, Middlesex HA1 3UJ

LAURENCE BERMAN, MRCP, FRCR, senior registrar, department of radiology LESLIE KLENERMAN, FRCS, consultant orthopaedic surgeon

Correspondence to: Dr L Berman, c/o Department of Radiology, Hospital for Sick Children, Toronto, Ontario, Canada. the Barlow test has been considered to be potentially harmful. Despite the introduction of testing for instability the incidence of established dislocation in both the United Kingdom and elsewhere has not decreased. ${ }^{+6}$ One series showed the incidence to have trebled since the introduction of screening. ${ }^{4}$

During the three years that hip ultrasound has been practised at this hospital it has become clear that a dislocated or normal hip is occasionally incorrectly diagnosed even by senior orthopaedic surgeons and paediatricians.

This study was undertaken in response to clear cases of clinical ambiguity and to evaluate whether anatomical abnormalities could be detected in clinically normal hips. We also tried to identify those unstable hips that might safely be left unsplinted.

Much of the early work on hip ultrasound was done by Graf, who described the ultrasound anatomy of the infant hip and devised a classification of four main types, ranging from normal to complete dislocation. ' Graf's study and those of other workers have recently been reviewed. ${ }^{8}$ Graf's classification is useful, and familiarity with it facilitates the understanding of this study.

Graf's type I (fig 1) is the normal appearance with a well formed bony acetabular roof and deep acetabular concavity. A small amount of hyaline cartilage is present at the lateral margin of the acetabulum, from which extends the labrum, encompassing the femoral head.

The type II hip has a much shallower bony acetabular concavity but has a larger hyaline cartilaginous component to the acetabular roof (fig 2). This would look dysplastic on a plain radiograph, but in the first few months of life the cartilage in the acetabular roof should ossify, and thus this becomes a normal type I hip. If ossification fails to occur this constitutes an acetabular dysplasia.

In the type III hip the labrum is actually displaced laterally by the femoral head (fig 3 ). The figure shows that the bony acetabulum is extremely shallow with virtually no covering of the femoral head. The type IV hip shows a large amount of displacement, with minimal contact between labrum and femoral head (fig 4).

\section{Patients and methods}

There are around 3500 deliveries each year at this hospital. Between November 1985 and February 1986 babies delivered consecutively in the maternity unit were scanned. Parents were informed of the study at antenatal attendance, and signed consent was obtained. Babies admitted to 
the neonatal intensive care unit weighing less than $2000 \mathrm{~g}$ were excluded. One thousand and one babies were examined during the study.

All scans were performed by one of us (LB) within 48 hours of delivery and were conducted on the postnatal wards using a Diasonics 100 scanner and a $7.5 \mathrm{MHz}$ sector transducer with direct skin contact. Coronal scans of the femoral head and acetabulum were obtained, and a permanent dry silver image of all examinations was retained. No form of restraining device was necessary, and all scans took less than one minute to perform. The ultrasonologist assigned the baby to one of three management categories based on the ultrasonic findings. These were: normal, Graf type Idischarge; intermediate, type II-rescan in three months to confirm ossification of acetabulum; and abnormal, type III or IV-refer to surgeon.

The results of scanning were not considered to be abnormal merely on the basis of observed instability, although this was recorded. Even if the femoral head was seen to slip in and out of the acetabulum during the scan the child was not referred to the orthopaedic clinic, provided that, when spontaneously reduced, the hip assumed the normal Graf type I appearance.

Babies were examined by the paediatric senior house officer within 24 hours of delivery, and if dislocation or instability was suspected the child was
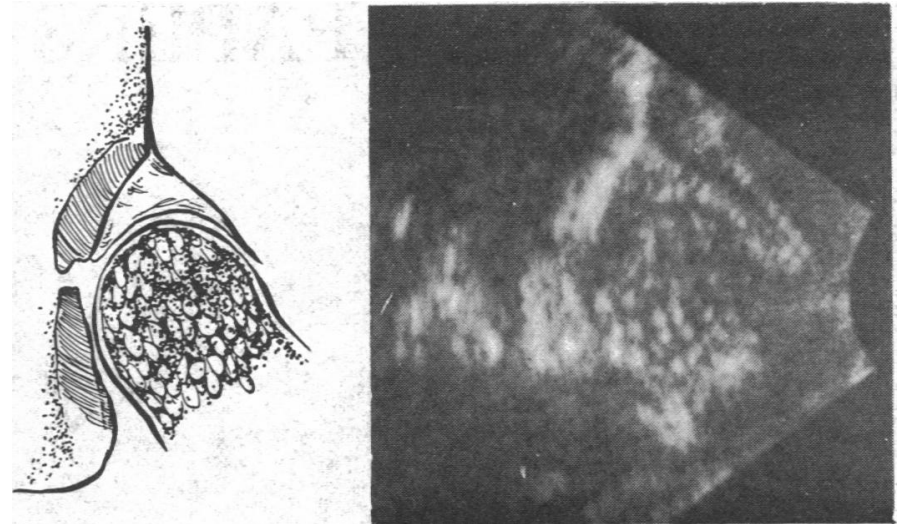

FIG 3-Graf type III hip with labrum deviated cephalad and minimal bony covering of femoral head (left hip of child shown in figs 5 and 6 ).

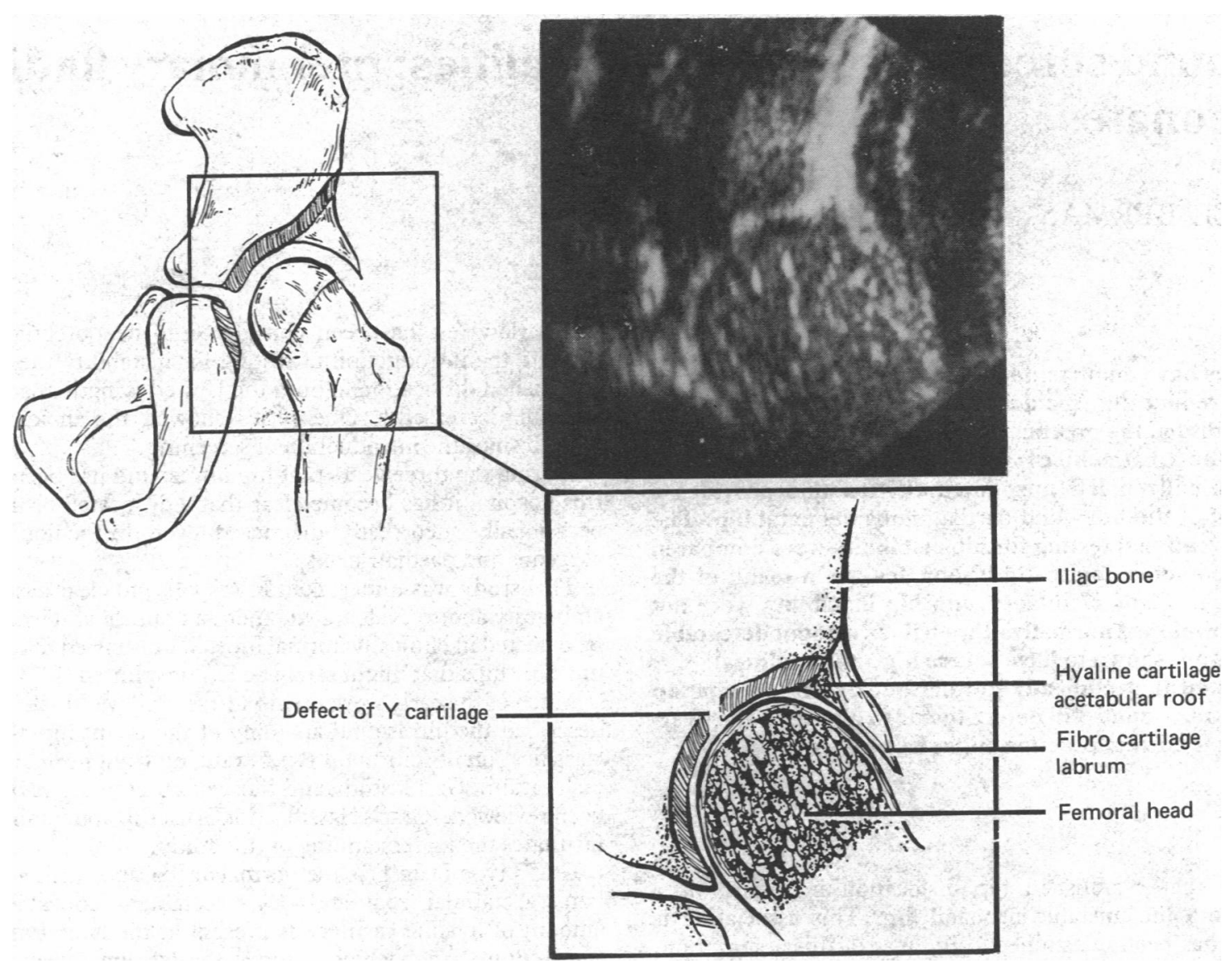

FIG 1-Graf type I hip: the normal neonatal appearance.

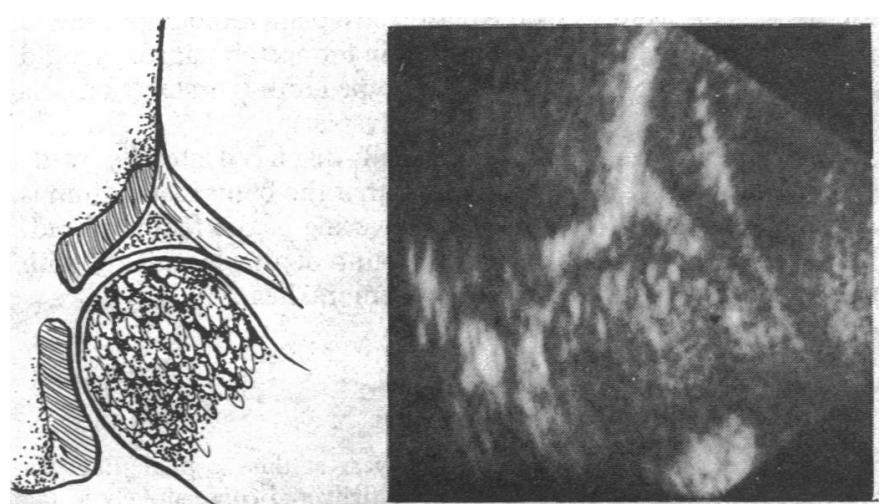

FIG 2-Graf type II hip: shallower bony acetabulum with larger cartilaginous component to acetabular roof. re-examined by the paediatric registrar. The paediatricians and ultrasonologists were unaware of each other's findings. All babies with suspected abnormalities, whether from clinical or sonographic findings, were seen by one consultant orthopaedic surgeon (LK) before mother and child left the hospital.

In accordance with existing practice certain clinically normal babies considered to be at high risk of hip abnormality were also referred to the consultant surgeon by the paediatricians. These risk factors included birth by extended breech delivery, a history of hip dislocation in a sibling or parent, and other orthopaedic problems such as clubfoot. No babies were discharged from follow up by the surgeon, whatever the reason for referral, until a radiograph of normal, ossified hips had been obtained.

In all cases the orthopaedic surgeon was unaware of the source of referral-that is, whether from paediatrician or ultrasonologist, or both. $\mathrm{He}$ was told the side of the suspected abnormality and whether the baby was clinically normal but in a high risk category. After completing his examination and formulating a treatment plan the surgeon was informed of the paediatrician's and ultrasonologist's assessments. 

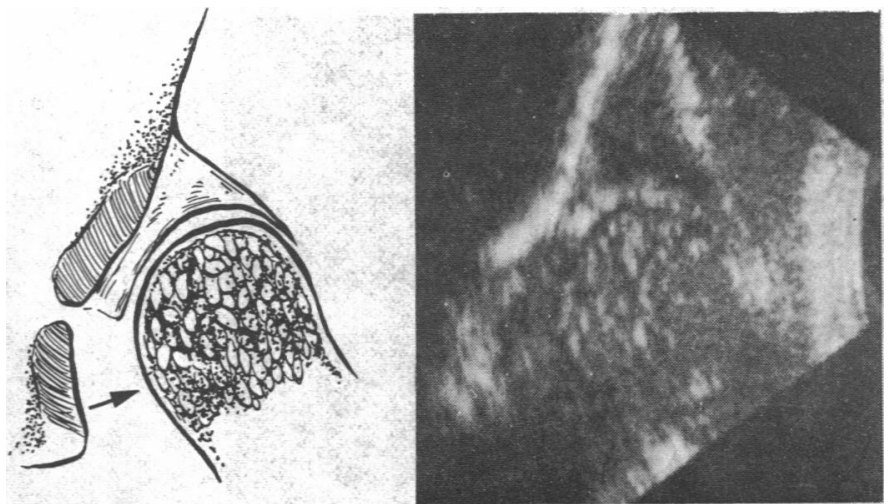

FIG 4-Graf type IV hip: large amount of lateral and cephalad movement of femoral head. been discharged from the orthopaedic clinic with normal radiographs showing ossified hips. This included 14 of the 17 infants referred by the paediatricians because of hip instability. The ultrasonologist's five initial referrals included three babies with unstable hips, which, although spontaneously reducible, assumed the Graf type II appearance with an unossified lateral acetabular margin. These three babies were also in the group of 17 referred by the paediatricians because of instability.

The two other babies referred by the ultrasonologist had no clinical signs but had ultrasound evidence of an extremely abnormal shallow acetabulum with an inadequately covered femoral head displacing the labrum cephalad, the Graf type III hip. Both these babies had plain radiographs which supported the ultrasonic findings and were still radiologically abnormal at the time of writing (figs 5 and 6 ).

Twenty three $(2 \cdot 3 \%)$ babies were recalled by the ultrasonologist for rescanning at 2-4 months because of an initial finding of a Graf type II hip with unossified lateral acetabular margin. Twenty two had assumed the normal Graf I appearance. One still had extremely shallow acetabula, although there was no evidence of hip displacement. This was confirmed by

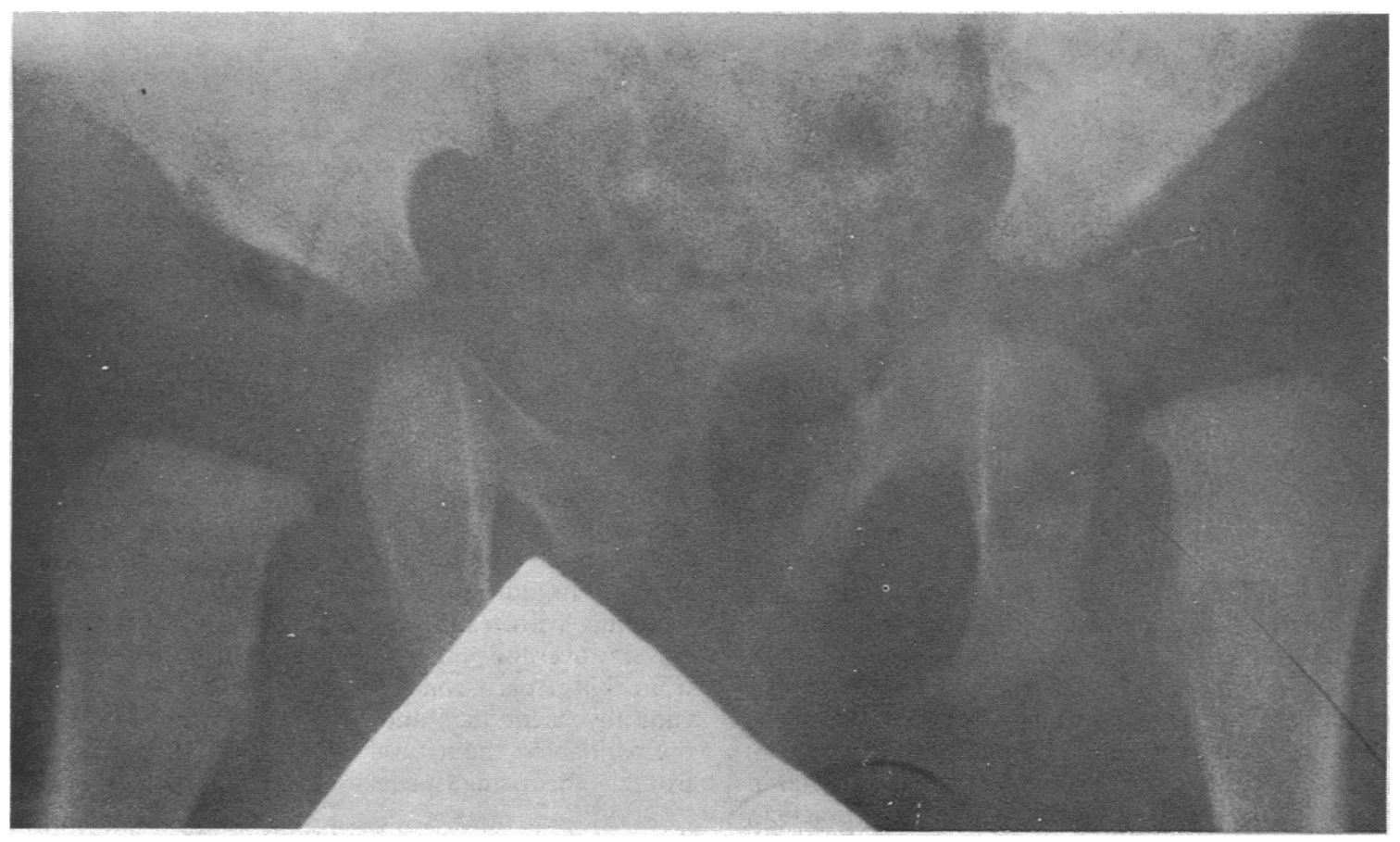

FIG 5-Baby D at birth: no clinical abnormality detectable (ultrasonic image shown in fig 3).

Only babies referred by the ultrasonologist underwent radiography as neonates. This was undertaken to compare the ultrasound impression of structural abnormality-for example, shallow acetabulum or displaced femoral head-with accepted plain radiological criteria such as increased acetabular angle. All the others who were referred underwent radiography at 2-4 months of age.

In view of the orthopaedic surgeon's and ultrasonologist's familiarity with ultrasound images of the hips, it was decided that hips with normal scans would not be splinted even if obviously clinically unstable but would be reexamined and rescanned at 3 weeks of age. Treatment would be started if instability was still detected at this stage. All babies in this study will be examined at 2-3 years of age to confirm normal development.

\section{Results}

One thousand and one babies were scanned. The paediatricians referred $45(4.5 \%)$ infants to the orthopaedic surgeon, compared with $5(0.5 \%)$ initial referrals from the ultrasonologist. Seventeen of the babies referred by the paediatricians had hip instability, 22 had had an extended breech delivery, five had a family history of hip dislocation, and one had clubfeet. Forty two of the 45 babies referred by the paediatricians yielded normal ultrasonic images and had been "discharged" by the ultrasonologist. All 42 developed normally without treatment, and at the time of writing most had plain radiography. As an incidental finding, three babies $(0.3 \%)$ had sonographically detectable ossific nuclei at birth, shown as a nidus of bright echoes in the otherwise weakly echogenic femoral head.

\section{Discussion}

We attempted to define both the cartilaginous and bony anatomy of the hips of a large number of neonates. Much of this information would formerly have required the use of contrast arthrography. Studies using plain radiographs have been undertaken, ${ }^{9}$ but the use of conventional radiography for screening would be unacceptable. In addition, the pitfalls of interpreting radiographs of the unossified hip are well documented ${ }^{10}$ despite Ortolani's claim that these provide an adequate image. ${ }^{11}$

Leck has observed that as babies with unstable hips are almost invariably treated and most cases of established dislocation arise in babies considered normal at birth the clinical course of hip instability remains an enigma. ${ }^{1}$ From data collected before screening was introduced it is known that unstable neonatal hips are much more common than eventual dislocation in unscreened populations. The incidence of unstable hips in this study (1.7\%) agrees closely 


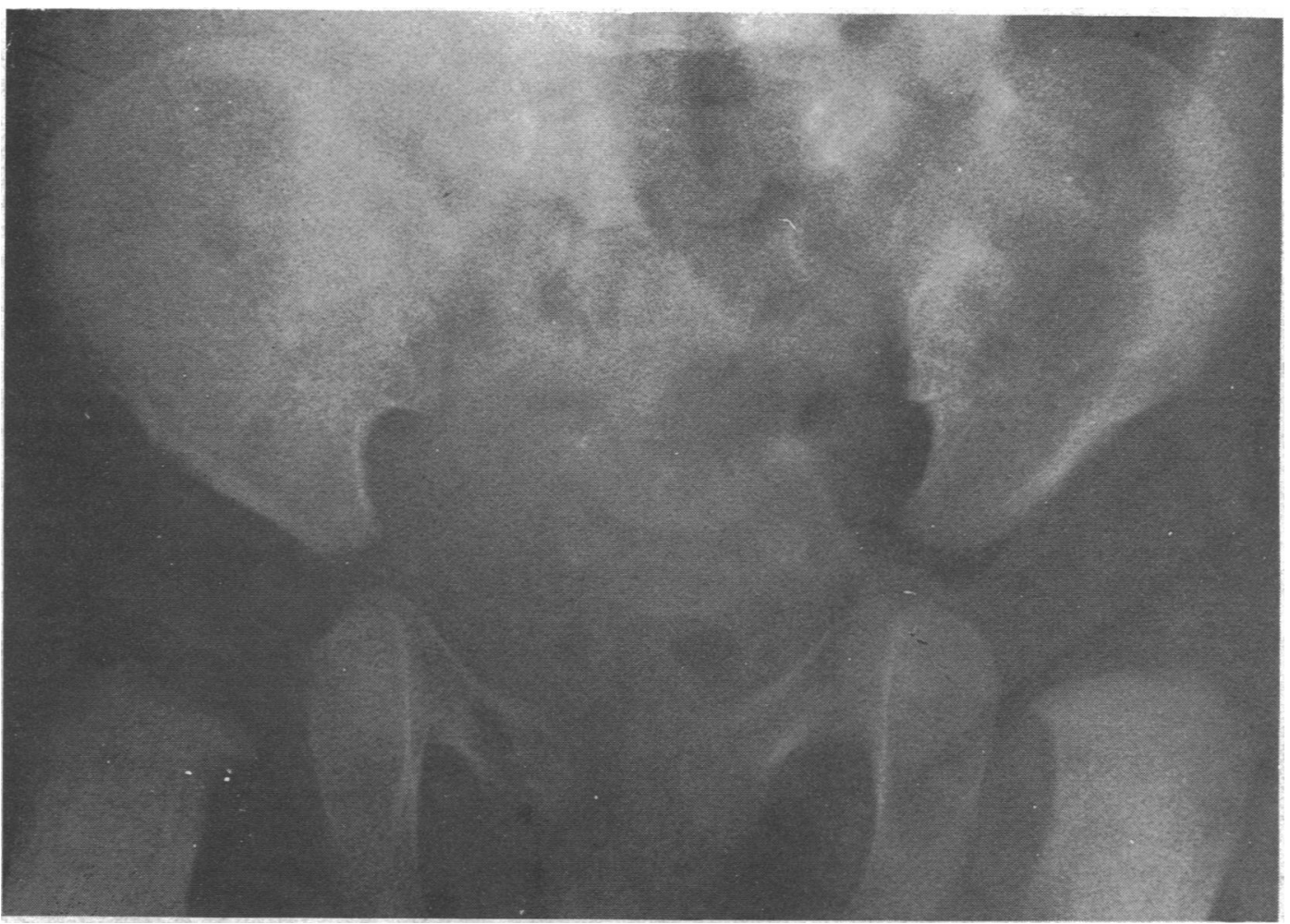

FIG 6-Baby D aged 4 months after treatment in Pavlic harness. Still no detectable clinical abnormality.

with that in many others. Leck estimated that in a recent series where all unstable hips were splinted ${ }^{12} 22$ babies may thus have been treated for each one who actually required splinting. ${ }^{1}$

There are three possible reasons why screening has failed to eradicate established dislocation. Firstly, neonatal instability may be overlooked at birth by an inexperienced clinician. Secondly, there may be a clinically silent predislocation stage based on an anatomical abnormality (such as a shallow acetabulum) which progresses to dislocation when weight bearing begins (this view is entertained by some ${ }^{23}$ but vigorously opposed by others. ${ }^{9}{ }^{13}$ ) Thirdly, established dislocation may be a condition acquired later in infancy; in support of this the danger of certain nursing practices such as swaddling babies in a position of leg adduction and extension has been emphasised. ${ }^{14}$ The only statistic concerning the prevalence of hip abnormality that is beyond question is the incidence of established dislocation. Why this incidence appears to be rising ${ }^{46}$ remains a mystery, but one theory is that screening manoeuvres actually predispose to late dislocation.

It is not claimed from these results that ultrasound screening will reduce the incidence of established hip dislocation. This will necessitate both the long term follow up of the 995 infants deemed normal and a study of a larger population of neonates. Several useful points can, however, be made. Firstly, hips may be clinically stable but anatomically abnormal. In figs 3, 5, and 6 the left hip is not normal despite treatment in a Pavlic harness. The femoral head is concentrically placed in an extremely deficient acetabulum. If this is the sort of abnormality that progresses to dislocation when weight bearing begins then it is possible that early detection will not improve the child's prognosis. Secondly, all 42 babies referred by the paediatricians but considered normal by the ultrasonologists developed normally. This includes 14 with unstable hips who were not treated because an ultrasound examination at birth yielded normal results, suggesting that the unstable hips that might safely be left unsplinted were identified. Thirdly, if cases of dislocation eventually arise in the sonographically normal group this will be a convincing argument that hips both stable and anatomically normal at birth can progress to established dislocation; however, this is not yet known. Fourthly, in no instance did the plain radiograph provide any additional information or contradict the interpretation of the ultrasonic image. The traditional lines and angles protracted on the plain radiograph should be replaced by the routine use of ultrasound for imaging the unossified hip.

This pilot study and the persisting incidence of established dislocation indicate that a larger study comparing ultrasound screening with testing for instability should be performed. Controversy over the potential hazards of ultrasound should not detract from this. Apart from the possibility of detecting abnormal but clinically silent hips and avoiding the unnecessary splinting of normal babies, the natural history of this condition might emerge from the theorising, speculation, and anecdote that prevail.

We thank Diasonics-Sonotron for generous support of this project.

\section{References}

1 Leck I. An epidemiological assessment of neonatal screening for dislocation of the hip. $\mathcal{f} R$ Coll Physicians Lond 1986;20:56-62.

2 Wilkinson JA. Congenital displacement of the hip joint. Berlin: Springer-Verlag, 1985.

3 Cyvin KB. Congenital dislocation of the hip joint. Clinical studies with special reference to

pathogenesis. Acta Paediatr Scand (Suppl) 1977;263:1-67.
4 Catford JC, Bennett GC, Wilkinson JA. Congenital hip dislocation: an increasing and still Catford JC, Bennett GC, Wilkinson JA. Congenital
uncontrolled disability. $B r$ Med f 1982;285:1527-30.

uncontrolled disability. $B r M e d \mathcal{F} 1982 ; 285: 1527-30$.
5 Klingberg MA, Chen R, Chemke J, Levin S. Rising rates of congenital dislocation of the hip. Lancet 1976; 298.

6 Bjerkdal T, Bakketeig LS. Surveillance of congenital malformation and other conditions of the newborn. Int $\mathcal{F}$ Epidemiol 1975;4:31.

7 Graf R. Classification of hip joint dysplasias by means of ultrasonography. Arch Orthop Trauma Surg 1984;102:248-55.

8 Berman L, Catterall A, Meire HB. Ultrasound of the hip: a review of the applications of a new technique. Br f Radiol 1986;59:13-7.

9 Caffey J, Ames R, Silverman WA, Ryder CT, Hough G. Contradiction of the congenital dysplasia - predislocation hypothesis of congenital dislocation of the hip through a study of the normal variation in acetabular angles at successive periods in infancy. Pediatrics 1956;17: $632-40$.

10 Blank E. Some effects of position on the roentgenographic diagnosis of the infant hip. Skeletal Radiol 1981;7:59-61.

11 Ortolani M. Detecting the dislocated hip. Lancet 1978;i:506-7.

12 Dunn PM, Evans RE, Thearle MJ, et al. Congenital dislocation of the hip: early and late diagnosis and management compared. Arch Dis Child 1985;60:407-14.

13 Barlow TG. Early diagnosis and treatment of congenital dislocation of the hip. $f$ Bone foint Surg 1962;48B:292-301.

14 Salter RB. Etiology, pathogenesis and possible prevention of congenital dislocation of the hip. Can Med Assoc J 1980;98:933-45.

(Accepted 3 fuly 1986) 